\title{
Development of Ultrasonic Modulation Probe for Fluorescence Tomography Based on Acousto-Optic Effect
}

\author{
Trinh Quang Duc, Shunsuke Kaneta, and Masaki Kobayashi \\ Department of Electronics, Graduate School of Engineering, Tohoku Institute of Technology, 35-1 Yagiyama-Kasumi cho, Taihaku-ku, \\ Sendai 982-8577, Japan \\ Correspondence should be addressed to Trinh Quang Duc, trinhduc@tohtech.ac.jp
}

Received 24 December 2010; Revised 4 April 2011; Accepted 30 May 2011

Academic Editor: Jianan Qu

Copyright ( $) 2011$ Trinh Quang Duc et al. This is an open access article distributed under the Creative Commons Attribution License, which permits unrestricted use, distribution, and reproduction in any medium, provided the original work is properly cited.

\begin{abstract}
We have developed an ultrasonic probe for fluorescence modulation to image fluorescence within biological tissues. The probe consists of a focused ultrasonic transducer mounted on actuators for mechanical fan scanning, which can be used in contact with the measuring object aiming for clinical application. The mechanical fan scanning employed in the probe has a beneficial feature of portability. As a result, fluorescent beads, which were localized with the diameter of $2 \mathrm{~mm}$ at $20 \mathrm{~mm}$ depth in a pork meat tissue, were detected with resolution of $3 \mathrm{~mm}$. The system performance denotes the feasibility of development towards the final goal of ultrasonic fluorescence modulation tomography for clinical applications.
\end{abstract}

\section{Introduction}

Fluorescent labeling technique has become an effective tool for life science research. Numerous research applications to explore biological function have been developed [1-5]. However, the characteristics of light scattering in biological tissues limit the use of optical methods for the investigation of fluorescence in biological system [6]. For clinical application, detection of the sentinel lymph node using fluorescent substances is expected for diagnosis of the cancer metastasis. For this purpose, detection of the localized fluorophore which is normally located in $10-20 \mathrm{~mm}$ depth from the body surface is required with a few millimeters resolution. For the fluorescence imaging in biological tissue, some advanced techniques such as continuous-wave fluorescence diffuse tomography [7], time-domain fluorescence diffuse tomography [8], and frequency-domain fluorescence diffuse tomography [9] have been proposed for the application of detecting fluorescence in small animals. These methods are based on modeling of photon transport in tissue and reconstruct the image from the obtained data through inverse problem. However, they require large amounts of computation. On the other hand, a method using ultrasound, which assists extracting optical absorption based on the speckles modula- tion, has been demonstrated [10]. Although the fluorescence emission in biological tissue is incoherent and considered that the modulated signal is much weaker than the speckle modulation $[10,11]$, we demonstrated the fluorescence imaging with a narrow-band detection of modulated signal [12]. The mechanism of fluorescence modulation with ultrasonic waves is based on variation of refractive index and scattering coefficient $[12,13]$ as well as density of fluorophore [14] under interaction of the sound field. The mod ulated fluorescence signal depends on sound pressure in the ultrasonic focal region. By scanning the ultrasonic focus, the position of localized fluorophore in the tissue is determined. This technique also considerably improves the imaging of fluorescence in thick tissue $[12,13]$ which can be applied for detection of the sentinel lymph node. In the former report [12], a turbid media formed by agarose gel with intralipid containing a fluorescent object was used to demonstrate fluorescence imaging of the sample. Tomographic images were obtained under the condition of immersion in a water tank through mechanical raster scanning of the concave type ultrasonic transducer which was equipped to the water tank.

To apply this technique in practical use, we have been developing a portable probe which can be used in contact with the body without using the water tank. To achieve 


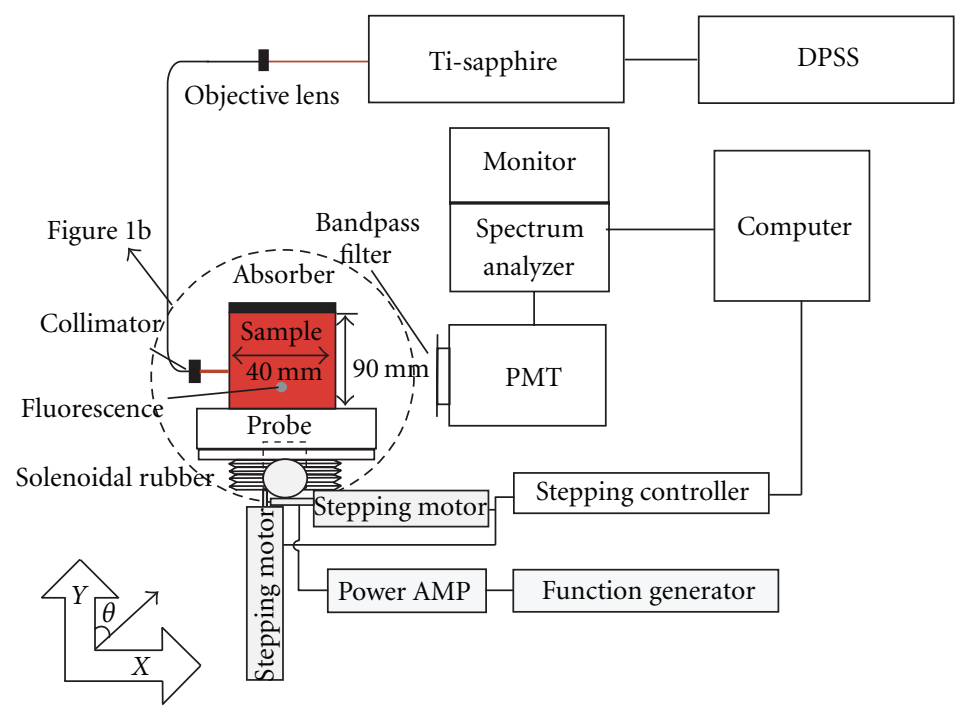

(a)

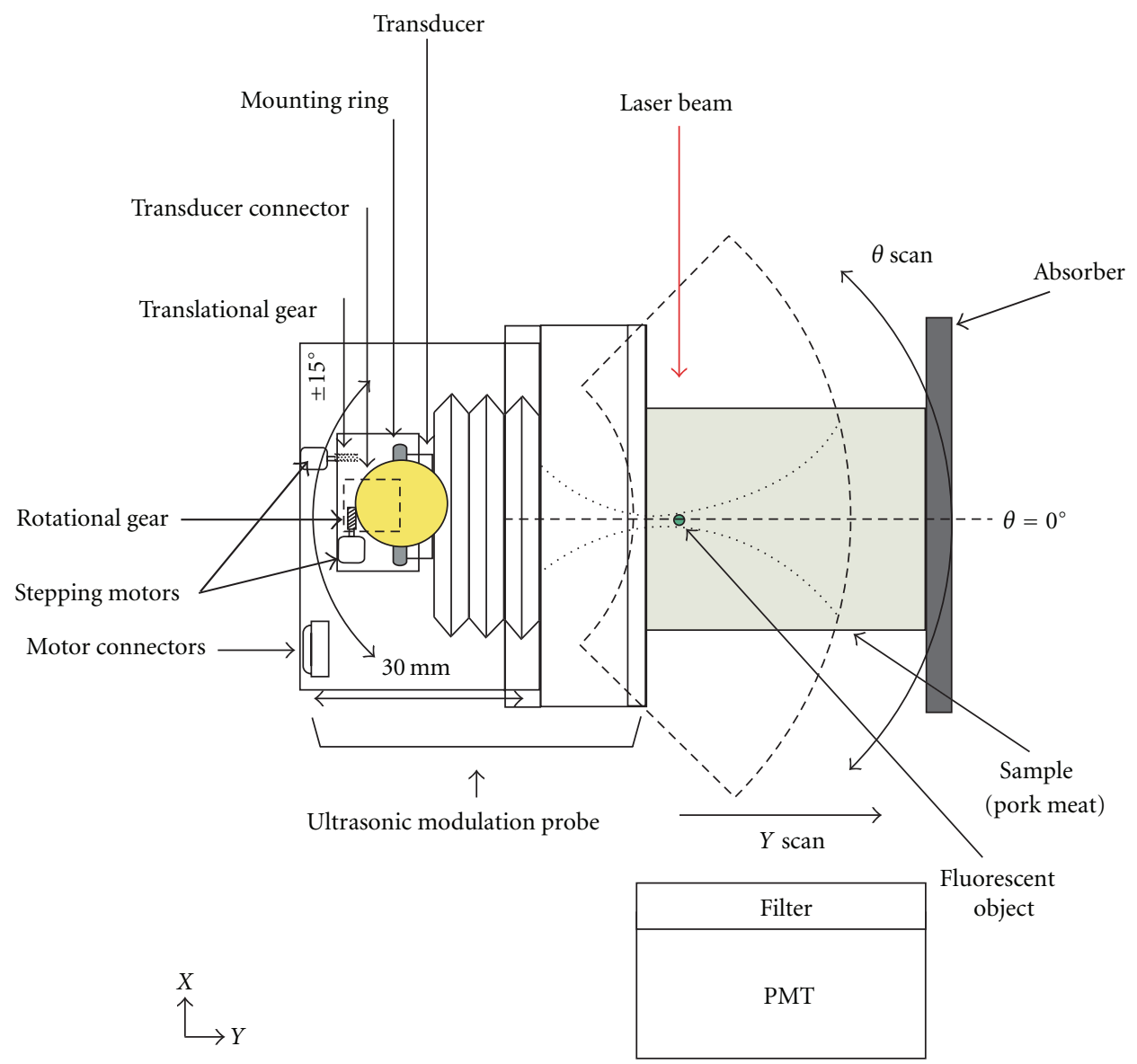

(b)

Figure 1: (a) Schematic of experimental setup. A tissue sample which includes a buried fluorescent object is placed on an adjustable plate. Probe is the combination of ultrasonic transducer with rotational and translational motion. (b) Structure diagram and operating principle of the ultrasonic modulation probe. 


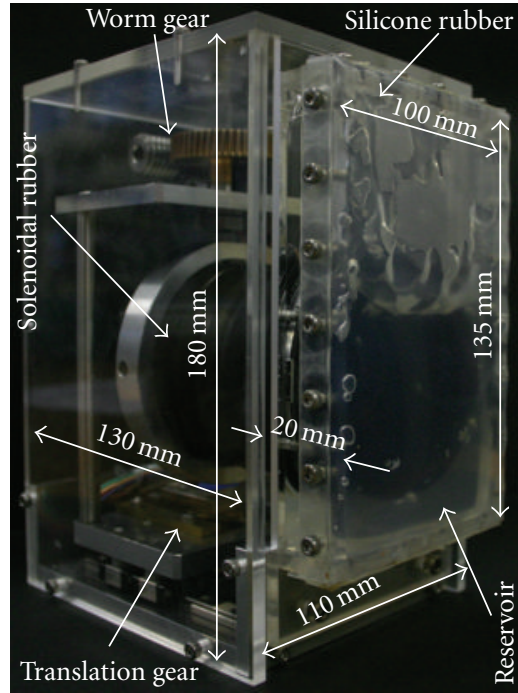

(a)

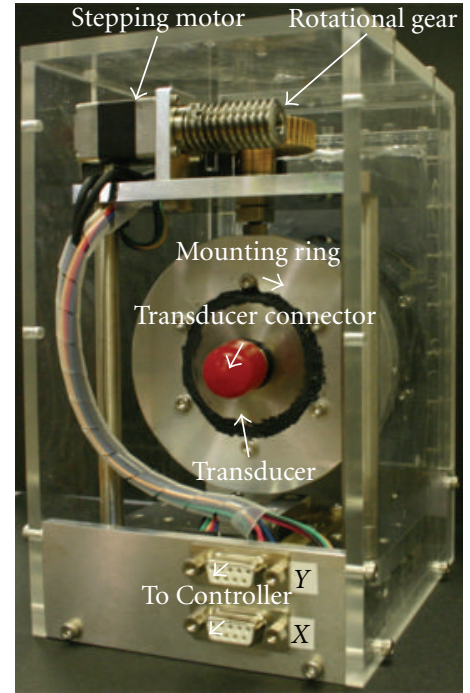

(b)

Figure 2: (a) The front view of the probe. Mounting ring holds the ultrasonic transducer to mount it on rotational mechanism. The rotational and translational motions are actuated by worm gear and translational gear. (b) The back view of the probe.

the portable probe for clinical application, the probe system, which can modulate the fluorescence with direct contact to the tissue surface, have to be investigated and examined in comparison to the former system. For the purpose, we first tried to use a phased-array ultrasonic transducer which can be controlled with electronic scanning and studied [15]. However, the discreteness of the transducer elements to form the focus by the phased-array system leads to generation of the artifact in fluorescence image. The phased-array ultrasonic transducer seems to be not suitable for fluorescence modulation. To overcome this obstacle, the concave type ultrasonic transducer which provides a continuous acoustic wave with high stability was considered. Resonant frequency of $1 \mathrm{MHz}$, which determines the focal region diameter measuring $3 \mathrm{~mm}$ as imaging resolution, was used in consideration of the performance of ultrasonic penetration and appropriate resolution for clinical application to detect sentinel lymph node. The probe equipped with this transducer was designed with its mechanical scanning configuration containing ultrasound conductive media inside. For scanning performance, an appropriate mechanism was considered with the following requirements. The focal point of the acoustic wave can be scanned throughout a plane of the target. The working area of the transducer should be minimal while scanning. From these requirements, fan scanning of the ultrasonic transducer by two-motion mechanism was adopted for the probe. This paper describes the design of the developed probe and demonstrates the imaging of a fluorescent object embedded in a pork meat.

\section{Materials and Methods}

Figure 1(a) shows the experimental setup. For excitation, we used a continuous-wave light at wavelength of $720 \mathrm{~nm}$ generated by a Ti:sapphire laser system (Coherent 899-01) pumped with a diode-pumped solid-state (DPSS) laser system (Coherent Verdi V-6). The laser beam is guided by an optical fiber and collimated to $1 \mathrm{~mm}$ diameter to illuminate the tissue sample. A photomultiplier tube (PMT) with a band-pass filter, which is located at the opposite side of the incident point of the laser beam, is used as a detector (R375, Hamatsu Photonics). The probe which is incorporated with an ultrasonic transducer (V392-SU; Olympus-NDT, focal length: $60 \mathrm{~mm}$, transversal focus size: $2.5 \mathrm{~mm}$ ) has contact with the tissue sample on the plane which is perpendicular to the plane of the laser incidence. Ultrasonic continuous wave from the transducer is generated by a function generator through a power amplifier. Direction of the laser beam $(X$ axis) is perpendicular to the ultrasound beam ( $Y$-axis). Fluorescence signal from the PMT is fed into a spectrum analyzer with band width of $500 \mathrm{~Hz}$ at the excitation frequency of the ultrasonic transducer.

The probe, comprising ultrasonic transducer, actuators, mounting system, and reservoir for ultrasonic gel, was designed as shown in Figure 1(b). Pictures of front view and back view of the probe with indication are present in Figure 2. The focused ultrasonic transducer was driven by a $1 \mathrm{MHz}$ continuous sinusoidal wave with amplitude of $90 \mathrm{~V}$ to generate a pressure approximate $6 \times 10^{4} \mathrm{~Pa}$ at the focus region (10 $\mathrm{mm}$ on longitudinal with $2.5 \mathrm{~mm}$ transversal). In the probe, two stepping motors with minigear systems as actuators are incorporated to provide the mechanism for rotational and translational motion. Ultrasonic gel is added in a reservoir measuring $135 \mathrm{~mm}$ length, $100 \mathrm{~mm}$ width, and $5 \mathrm{~mm}$ thickness. As shown in the front view of the probe (Figure 2(a)), the side of the reservoir is covered by a $0.5 \mathrm{~mm}$ thick silicone rubber sheet to make contact with the tissue sample, and a solenoidal rubber tube with $80 \mathrm{~mm}$ diameter 


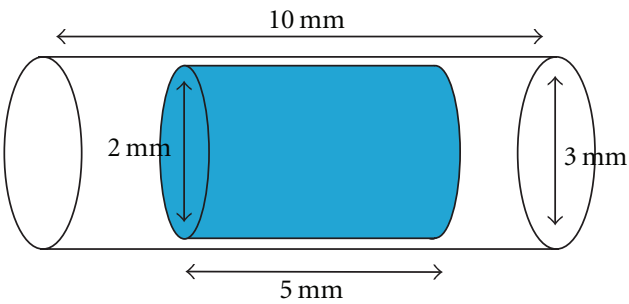

(a)

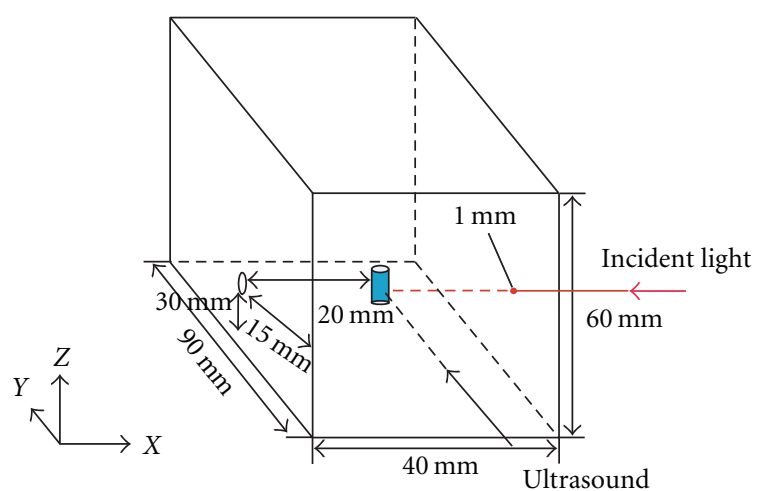

(b)

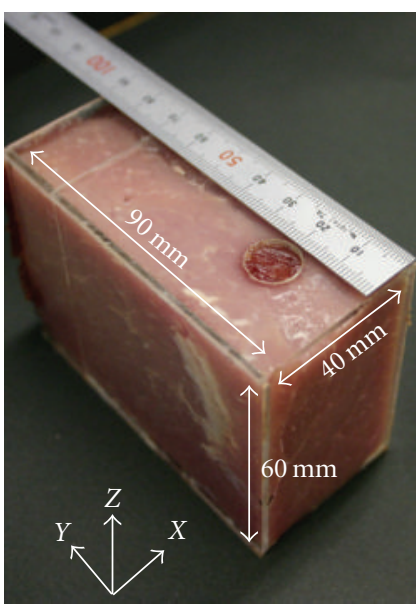

(c)

Figure 3: (a) Illustration of the fluorescent object. The fluorescent region was molded in a cylindrical silicone tube. Both heads of it were sealed with silicone gel. (b) Schematic of the tissue sample showing the buried position of the fluorescent object. Incident direction of excitation light beam and ultrasonic beam are indicated. (c) Appearance of the sample in an acrylic plastic case.

is used to allow the rotational and translational motion of the transducer. The back view of the probe is shown in Figure 2(b).

For scanning, the actuators are driven by computer through 2 channels of a stepping motor controller with minimum resolution of $0.01 \mathrm{~mm}$ and 0.1 degrees for translational and rotational motions, respectively. The maximum range of translational scanning ( $Y$-axis) of ultrasound focus is 0 $30 \mathrm{~mm}$. The origin of the $Y$-axis is set at the silicone rubber surface. The maximum rotational scanning ( $\theta$ axis) range for the ultrasonic focus is \pm 15 degrees. The origin of the $\theta$ axis is the center of the rotation which is parallel with the $Y$-axis. In addition, a sound absorber is placed on the end of the tissue, opposite the probe to reduce acoustic reflection at the side which causes generation of standing wave.

Figure 3 shows the preparation of tissue sample and fluorescent object. For the fluorescent object, the fluorophore of carboxylate-modified microsphere (F8799; Invitrogen, USA), which has $715 \mathrm{~nm}$ peak absorption and $755 \mathrm{~nm}$ emission wavelength, was used, molded in columnar agarose gel ( $5 \mathrm{~mm}$ long, $2 \mathrm{~mm}$ diameter) at $50 \%$ volume concentration, and sealed in a silicone tube. The dimensions of the fluorescent object are shown in Figure 3(a). The fluorescent object was buried in pork meat that was used as the tissue sample. The size of this tissue was $40 \times 60 \times 90 \mathrm{~mm}$ and a plane of $40 \times 60 \mathrm{~mm}$ was the contact side to ultrasound source. The fluorescent object was buried at position of $30 \mathrm{~mm}, 20 \mathrm{~mm}$, and $15 \mathrm{~mm}$ from the top, the right, and the contact side, respectively, as illustrated in Figure 3(b). The tissue sample was inserted in an acrylic plastic case to hold the tissue sample as shown in Figure 3(c). The sample was scanned in the rotation range from -7.5 degrees to +7.5 degrees with resolution of 0.5 degrees and the translation range from 0 to $30 \mathrm{~mm}$ with resolution of $0.5 \mathrm{~mm}$. The laser light of $70 \mathrm{~mW}$ power was used for excitation to obtain each fluorescence image which requires 30 minutes for scanning.

At each scanned position, three parameters: $\theta$ position, $Y$ position, and intensity of the modulated signal, were recorded by a computer through GPIB ports. The image of fluorescence was reconstructed by conversion of $\theta-Y$ of cylindrical coordinate system to $X-Y$ of cartesian coordinate system. The image was smoothen by the $7 \times 7$ convolution matrix filter and displayed in three dimensions. 


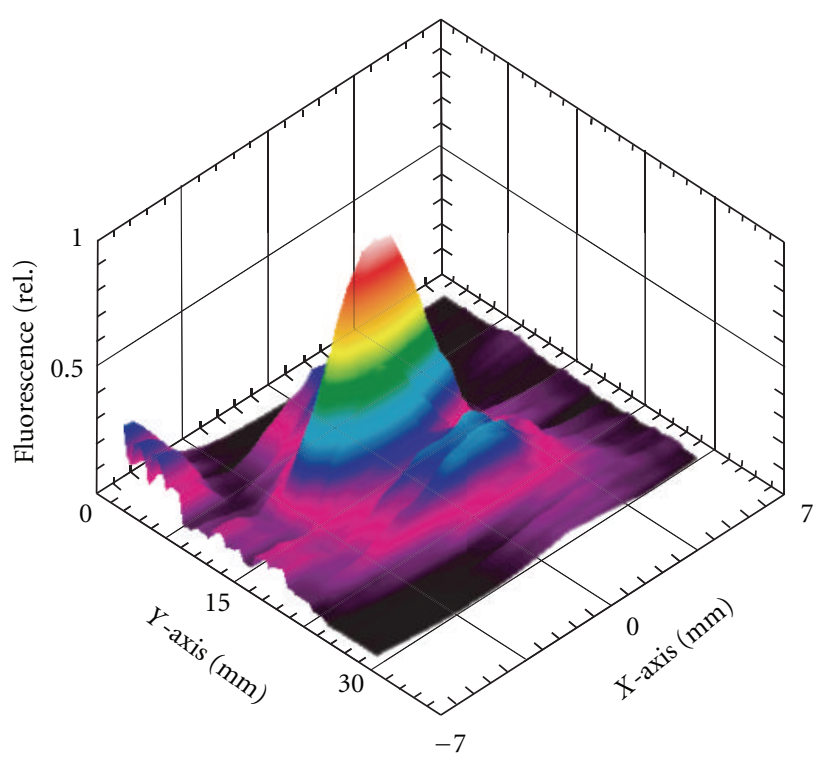

(a)

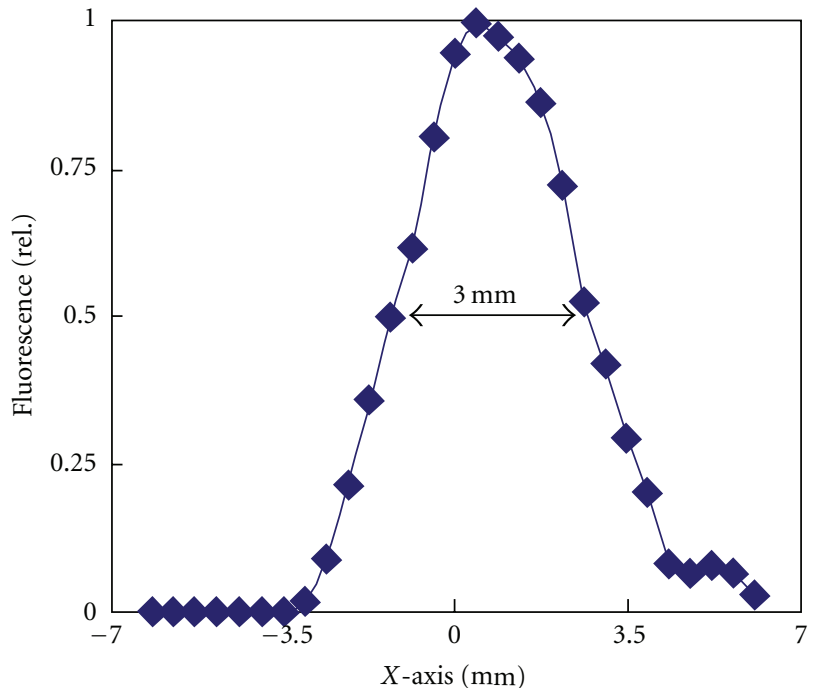

(b)

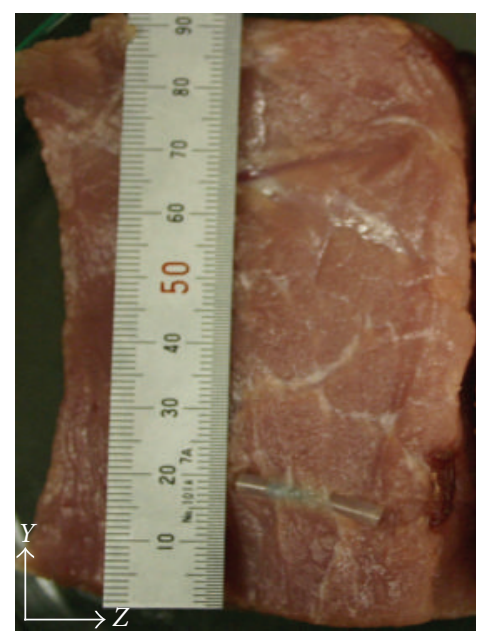

(c)

FIGURE 4: (a) The 2D cross-sectional image of fluorescent object within the tissue sample. (b) The $X$-profile of the image at peak point after using the threshold filter. (c) Picture of the section of the sample to show the buried fluorescent object.

\section{Results and Discussion}

Figure 4(a) shows a cross-sectional image of fluorescence. The signal level at the peak point is observed with $-97.5 \mathrm{dBm}$. Location of the peak, which is $15 \mathrm{~mm}$ on $Y$-axis, corresponds to the position of the fluorescent object embedded in the tissue sample. The profile of the peak along $X$ axis, sectioned at $15 \mathrm{~mm}$ on $Y$-axis, is shown in Figure 4(b), indicating the full width at haft maximum (FWHM) of approximate $3 \mathrm{~mm}$. The smaller peaks appearing near the main peak are believed to be the effect of the standing wave of ultrasound caused by the reflection at the back side of the tissue sample. Figure $4(\mathrm{c})$ is the picture of the tissue sample to show the cut along $Y$-axis at the position of the fluorescent object after the measurement. The averaged background noise, caused by electronic noise at $1 \mathrm{MHz}$, was $-108 \mathrm{dBm}$ in the measurement condition. To reduce the fluctuation of the background, in other words, to flatten the noise floor, the lower level of the threshold for the signal was set for image reconstruction. With taking into account of the transversal size of focus and convolution filter in image processing, the observed FWHM at the peak seems to be reasonable.

From these results, the developed probe has the performance to detect a localized fluorescent region of the size of $2 \mathrm{~mm}$ diameter with the resolution of $3 \mathrm{~mm}$, which is similar to the previous result obtained using the water tank system. To increase the imaging resolution, replacement of the ultrasonic transducer which has higher frequency is required. 
International Journal of Optics

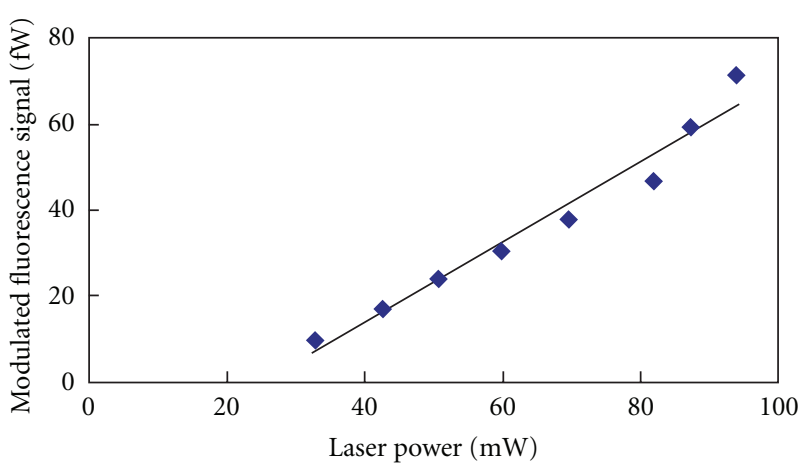

FIgURE 5: Excitation laser power versus modulated fluorescence signal quantified by the spectrum analyzer.

However, since the higher frequency of ultrasound restricts the penetration of the sound beam, the ultrasonic transducer used in the probe is suitable for the purpose to detect the sentinel lymph node.

The relationship between excitation power and modulated fluorescence signal at the center of fluorescent object is shown in Figure 5. The incident beam was increased from $30 \mathrm{~mW}$ to $100 \mathrm{~mW}$ in $10 \mathrm{~mW}$ steps, and the modulated fluorescent signal points are indicated in the graph. This intensity of the modulated fluorescent signal is obtained by the spectrum analyzer then converted from logarithmic $\mathrm{dBm}$ to linear scale with subtraction of the noise floor and fitted to the linear function. Modulated fluorescence intensity is linear to the excitation power. In this condition of the experiment, the lower limit of the detection of modulated fluorescence signal was determined to be $25 \mathrm{~mW}$ excitation from the cross point of the regression line to $X$-axis. These results appear to be consistent with the explanation of the mechanism of modulation in which the modulated intensity of fluorescence is proportional to the excitation.

\section{Conclusions}

An ultrasonic modulation probe for fluorescent tomographic imaging has been constructed and evaluated with imaging of a fluorescent object in a biological tissue using pork meat. We demonstrated the performance of this probe to image the fluorescent object at $20 \mathrm{~mm}$ depth in the pork meat tissue with $3 \mathrm{~mm}$ resolution as shown in the Figure $4(\mathrm{a})$ in a 2Dcross-sectional image. The mechanical fan scanning employed in the developed probe is beneficial to the fluorescence imaging of biological tissue as a portable probe, indicating the ability to apply it to the sentinel lymph node detection. To rebuild the tomographic images of fluorescence, another plane within the sample can be extracted by scanning along $Z$-axis which may be performed by manual or automatic mechanism with a $Z$-axis translation. Although the scanning was not carried out in this report, further setup with $Z$ scanning can be applied for that purpose. And for the future study to apply the probe for practical use, a combination of the light source and detector to the probe should be considered.

\section{Acknowledgment}

This work was supported by KAKENHI 19300162.

\section{References}

[1] W. B. Dandliker, R. J. Brawn, M.-L. Hsu et al., "Investigation of hormone-receptor interactions by means of fluorescence labeling," Cancer Research, vol. 38, pp. 4212-4224, 1978.

[2] P. Krist, L. Vannucci, M. Kuzma et al., "Fluorescent labelled thiourea-bridged glycodendrons," ChemBioChem, vol. 5, no. 4, pp. 445-452, 2004.

[3] T. C. Chu, F. Shieh, L. A. Lavery et al., "Labeling tumor cells with fluorescent nanocrystal-aptamer bioconjugates," Biosensors and Bioelectronics, vol. 21, no. 10, pp. 1859-1866, 2006.

[4] H. Theilen and W. Kuschinsky, "Fluorescence labeling of the capillary network in rat brains," Journal of Cerebral Blood Flow and Metabolism, vol. 12, no. 2, pp. 347-350, 1992.

[5] M. V. Marshall, J. C. Rasmussen, I.-C. Tan et al., "Near-infrared fluorescence imaging in humans with indocyanine green: a review and update," The Open Surgical Oncology Journal, vol. 2, pp. 12-25, 2010.

[6] J. Mobley and T. Vo-Dinh, "Optical properties of tissue," in Biomedical Photonics Handbook, T. Vo-Dinh, Ed., chapter 2, CRC Press, New York, NY, USA, 2003.

[7] R. B. Schulz, J. Ripoll, and V. Ntziachristos, "Experimental fluorescence tomography of tissues with noncontact measurements," IEEE Transactions on Medical Imaging, vol. 23, no. 4, pp. 492-500, 2004.

[8] A. T. N. Kumar, S. B. Raymond, A. K. Dunn, B. J. Bacskai, and D. A. Boas, "A time domain fluorescence tomography system for small animal imaging," IEEE Transactions on Medical Imaging, vol. 27, no. 8, Article ID 4449088, pp. 1152-1163, 2008.

[9] A. Godavarty, A. B. Thompson, R. Roy et al., "Diagnostic imaging of breast cancer using fluorescence-enhanced optical tomography: Phantom studies," Journal of Biomedical Optics, vol. 9, no. 3, pp. 488-496, 2004.

[10] L. Wang, S. L. Jacques, and X. Zhao, "Continuous-wave ultrasonic modulation of scattered laser light to image object in turbid media," Optics Letters, vol. 20, no. 6, pp. 629-631, 1995.

[11] G. D. Mahan, W. E. Engler, J. J. Tiemann, and E. Uzgiris, "Ultrasonic tagging of light: theory," Proceedings of the National Academy of Sciences of the United States of America, vol. 95, no. 24, pp. 14015-14019, 1998.

[12] M. Kobayashi, T. Mizumoto, Y. Shibuya, M. Enomoto, and M. Takeda, "Fluorescence tomography in turbid media based on acousto-optic modulation imaging," Applied Physics Letters, vol. 89, no. 18, Article ID 181102, pp. 1-3, 2006.

[13] M. Kobayashi, T. Mizumoto, Y. Shibuya, M. Enomoto, and M. Takeda, "Fluorescence tomography in turbid media based on acousto-optic modulation imaging," Applied Physics Letters, vol. 89, no. 18, Article ID 181102, 3 pages, 2006.

[14] B. Yuan, J. Gamelin, and Q. Zhu, "Mechanisms of the ultrasonic modulation of fluorescence in turbid media," Journal of Applied Physics, vol. 104, no. 10, Article ID 103102, 13 pages, 2008.

[15] Q. D. Trinh, Y. Nanbu, T. Suzuki, S. Takahashi, M. Takeda, and M. Kobayashi, "Fluorescence tomography based-on acoustooptic modulations with phased-array ultrasound transducer," in Photons Plus Ultrasound: Imaging and Sensing, vol. 7177-75 of Proceedings of SPIE, BiOS Part of SPIE Photonic West, San Jose, Calif, USA, January 2009. 

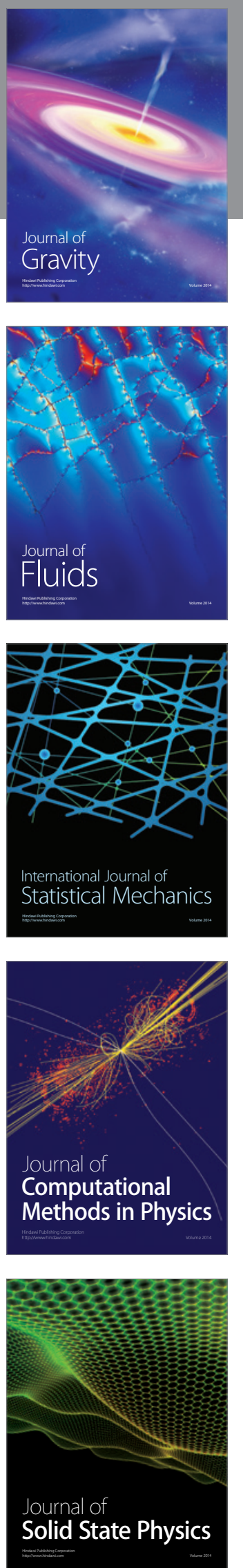

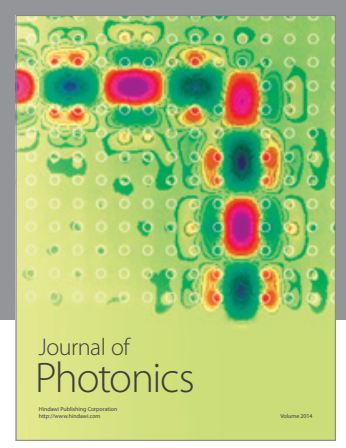

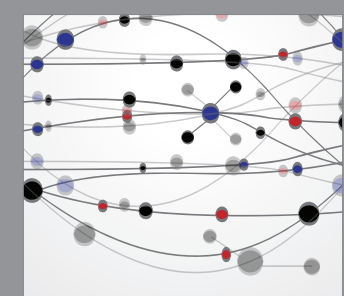

The Scientific World Journal
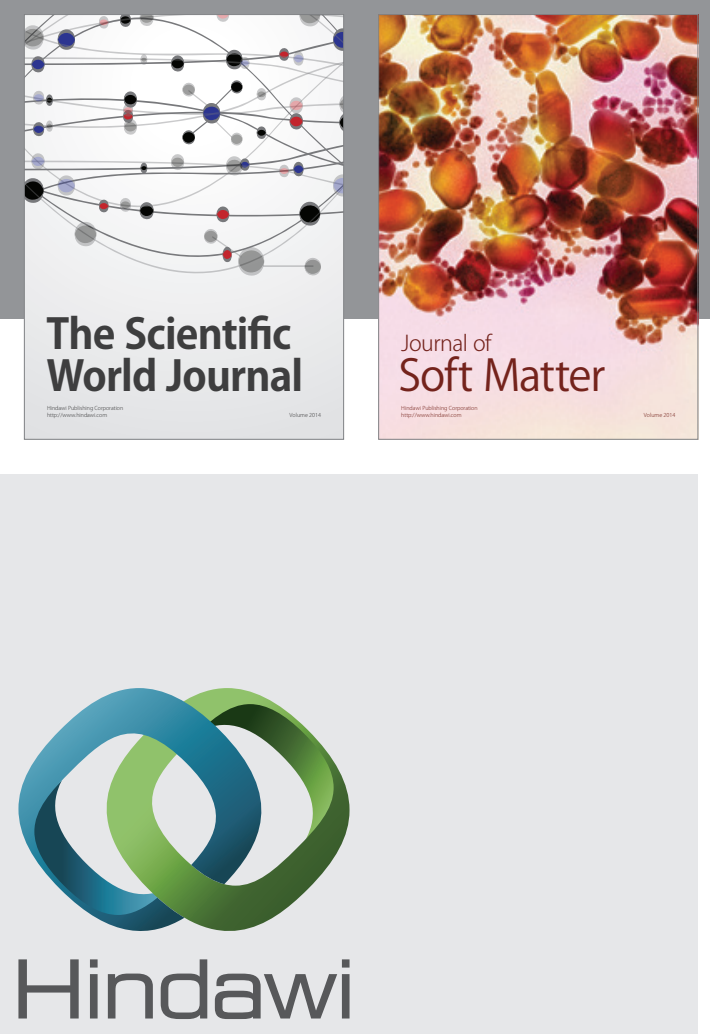

Submit your manuscripts at

http://www.hindawi.com
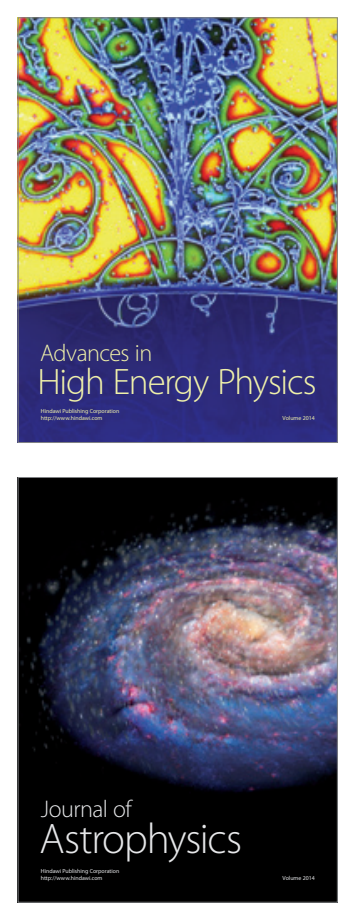
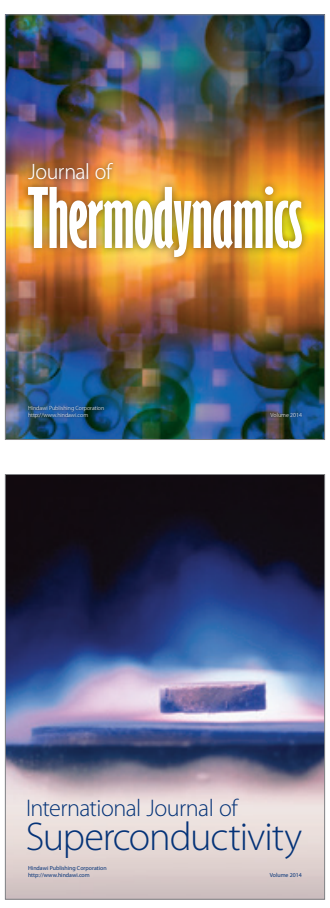
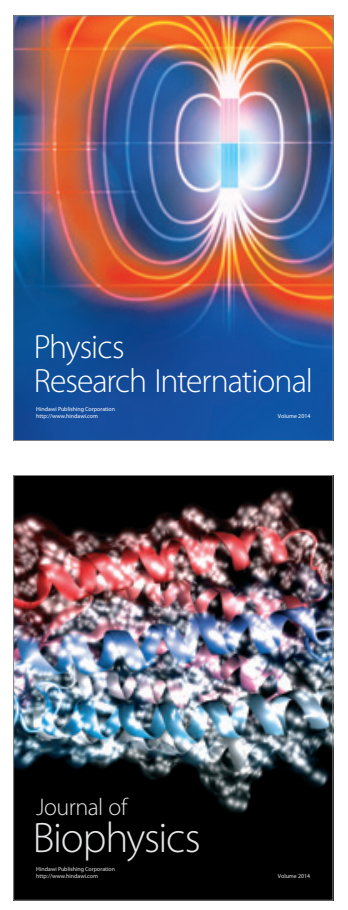
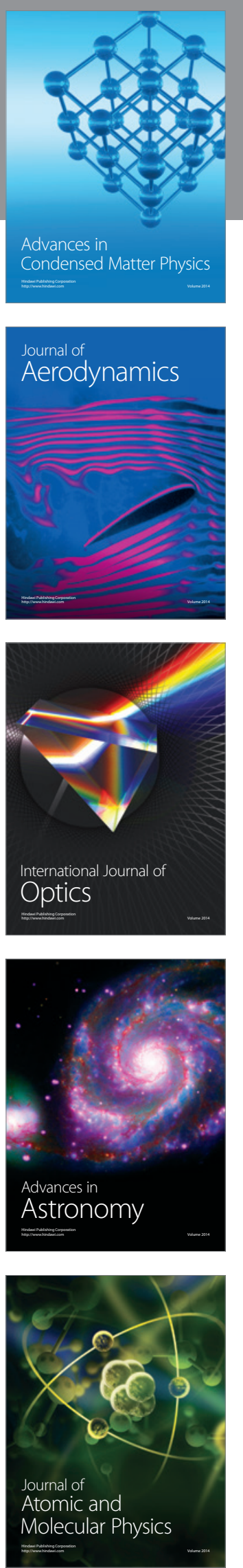